\title{
One-pot Synthesis of Arborescent Polystyrenes
}

\author{
Zhongshun Yuan and Mario Gauthier
}

Supporting Information

Solvent and Reagent Purification. Toluene (BDH, HPLC grade) and cyclohexane (EMD Chemicals, ACS Reagent) were purified by refluxing with oligostyryllithium under nitrogen, and introduced directly from the still into the reaction flask through poly(tetrafluoroethylene) (PTFE) tubing. Tetrahydrofuran (THF, Caledon, ACS Reagent) was refluxed with and distilled from sodium-benzophenone ketyl under nitrogen. Styrene (Aldrich, 99\%) was first distilled from $\mathrm{CaH}_{2}$ under reduced pressure, and further purified with phenylmagnesium chloride immediately before polymerization (Aldrich, $2.5 \mathrm{M}$ solution in THF; $1 \mathrm{~mL}$ of solution per $10 \mathrm{~mL}$ styrene, THF removed under vacuum before addition of styrene) and degassed with three freezingevacuation-thawing cycles before recondensation into an ampule with a PTFE stopcock under high vacuum. ${ }^{1}$ For the synthesis of analogous arborescent polystyrenes with different side chain lengths by the successive monomer addition method, styrene was diluted with THF to obtain a $10 \% \mathrm{v} / \mathrm{v}$ solution by condensing the dry solvent to the ampule under vacuum after monomer purification. 1,3-Diisopropenylbenzene (DIB, Aldrich, 97\%) was first purified by two distillations from $\mathrm{CaH}_{2}$ under vacuum. 1,4-Diisopropenylbenzene (1,4-DIB) was synthesized by the Grignard reaction of dimethyl terephthalate with $\mathrm{MeMgX}(\mathrm{X}=\mathrm{Br}, \mathrm{I})$ and dehydration by flash distillation from alumina at $250{ }^{\circ} \mathrm{C}$ under vacuum. ${ }^{2,3}$ The monomer was further purified by two vacuum distillations from $\mathrm{CaH}_{2}$. The two diisopropenylbenzene isomers were finally purified in an ampule by azeotropic drying with THF immediately before use, and mixed with purified styrene under nitrogen to obtain the desired monomer ratio in the mixture. After purification, all monomer ampules were stored at $-78{ }^{\circ} \mathrm{C}$ (dry ice) before use. The secbutyllithium solution ( $s$-BuLi, Aldrich, $1.3 \mathrm{M}$ in cyclohexane) was used as received; its exact concentration was determined to be $1.35 \mathrm{M}$ by the method of Lipton et al. ${ }^{4}$ Lithium chloride (Aldrich, 99.9\%) was flamed under high vacuum in an ampule and dissolved in dry THF by condensation under vacuum before use. The exact amounts of reagents employed in the reactions are specified in the examples provided in the Experimental Section.

Polymer characterization. Size exclusion chromatography (SEC) analysis was used to determine the apparent (polystyrene-equivalent) molecular weight $\left(M_{\mathrm{w}}, M_{\mathrm{n}}\right)$ and polydispersity index $\left(\mathrm{PDI}=M_{\mathrm{w}} / M_{\mathrm{n}}\right)$ of the graft polymer samples in routine work. The instrument used consists of a Waters 510 HPLC pump, a Jordi $500 \mathrm{~mm} \times 10 \mathrm{~mm}$ DVB linear mixed-bed column (linear polystyrene molecular weight range of $10^{2}-10^{7}$ ), and a Waters 410 differential refractometer (DRI) detector. THF at a flow rate of $1 \mathrm{~mL} / \mathrm{min}$ served as eluent, and linear polystyrene standards (molecular weight range of $1.25 \times 10^{3}-2.75 \times 10^{6}$ ) were used to calibrate the instrument.

The absolute molecular weight of some graft polymers was determined with a SEC system using a Wyatt Dawn DSP-F multiangle light scattering (MALLS) detector at $632.8 \mathrm{~nm}$. The SEC-MALLS system used consisted of a Waters 590 programmable HPLC pump and a Waters 2410 DRI detector for polymer concentration measurements (operation wavelength of $660 \mathrm{~nm}$ ). THF served as eluent at a flow rate of $1 \mathrm{~mL} / \mathrm{min}$. The G0 and G1 polymers were analyzed with a 
set of Waters $\mu$ Styragel columns $\left(7.8 \mathrm{~mm} \times 300 \mathrm{~mm}, 10^{4}, 10^{5}\right.$, and $10^{6} \AA$ pore sizes $)$ with a molecular weight range of $5 \times 10^{3}-10^{7}$. The G1 polymers were also analyzed using a set of two Polymer Laboratories PLgel $20 \mu \mathrm{m}$ MIXED-A ultrahigh molecular weight columns $(300 \mathrm{~mm} \times$ $7.5 \mathrm{~mm}$ ) with a linear polystyrene molecular weight range of $2 \times 10^{3}-4 \times 10^{7}$. Because of the significant band broadening observed for the Polymer Laboratories columns, band broadening correction $^{5}$ was applied to the analysis results. The refractive index increment values $(\mathrm{d} n / \mathrm{d} c)$ used in the light scattering calculations were measured in THF at $25{ }^{\circ} \mathrm{C}$ on a Brice-Phoenix differential refractometer equipped with a $632 \mathrm{~nm}$ band-pass interference filter. The effect of the second virial coefficient was not considered in the molecular weight calculations, which may have resulted in slightly $(<5 \%)$ underestimated molecular weight values. ${ }^{6}$

Viscosity measurements were made in toluene at $25.0 \pm 0.2{ }^{\circ} \mathrm{C}$ using a Cannon-Ubbelohde Size 75 capillary viscometer. Stock solutions of the polymers were prepared by dissolving $0.2-$ $1.0 \mathrm{~g}$ of polymer (depending on the sample) in $25 \mathrm{~mL}$ of solvent. The concentration of each stock solution was adjusted to give a flow time roughly double that of the pure solvent. The solutions and the pure solvent were filtered through $5 \mu \mathrm{m}$ PTFE membrane filters before the measurements. Series of five consistent $( \pm 0.2 \mathrm{~s})$ flow times were obtained at seven different concentrations, using successive solvent additions to the viscometer reservoir. The intrinsic viscosity of the polymers was determined by linear extrapolation of plots of $\eta_{\mathrm{sp}} / c$ versus concentration.

Reacting Species and Reactivity. The reaction of 1,3-diisopropenylbenzene (DIB) and 1,4diisopropenylbenzene (1,4-DIB) in cyclohexane with a 10-fold excess of $s$-BuLi was investigated under ambient conditions by Lutz et al. ${ }^{3}$ The two double bonds of DIB were almost isoreactive towards the highly reactive (non-selective) organolithium compound used in the reaction. Adduct formation was approximately $30 \%$ faster for the first double bond of 1,4-DIB, however. Another investigation of the same reaction under different conditions ( 2 equiv $s$-BuLi in toluene at $50{ }^{\circ} \mathrm{C}$ ) showed that a mixture of mono- and dilithiated adducts, as well as multilithiated oligomers were obtained initially, and that depolymerization of the oligomers occurred once a monomer (styrene or 1,1-diphenylethylene) was added to the reaction. ${ }^{7}$

Unfortunately, only fragmentary data is available in the literature on the anionic homo- and copolymerization of DIB and 1,4-DIB. The homopolymerization of both isomers is reversible albeit the exact values of their ceiling temperatures are unknown, and values have been suggested ranging from room temperature ${ }^{8}$ to around $50{ }^{\circ} \mathrm{C}{ }^{7}$ The homopolymerization of DIB apparently proceeds without significant reaction of the second isopropenyl moiety when monomer conversion is maintained below ca. $50 \%,{ }^{8}$ presumably due to the lower reactivity (and greater selectivity) of the DIB anionic propagating centers as compared to $s$-BuLi. In spite of the lower reactivity of the pendent isopropenyl moieties towards the propagating centers, the molecular weight distribution (MWD) of the homopolymer broadens rapidly and gelation is even observed at long reaction times. The anionic copolymerization of DIB has been performed with both $\alpha$-methylstyrene and styrene. ${ }^{7,8}$ For styrene and DIB, a strong tendency towards sequential polymerization of the two monomers was inferred from the color change (from yellow for polystyryl anions to dark brown for polyDIB anions) observed during batchwise copolymerization reactions, and the fact that the polymers obtained at low conversion were devoid of unsaturation. The copolymerization of DIB with $\alpha$-methylstyrene was shown to proceed in a more random fashion, the color remaining dark brown throughout the reaction, albeit with preferential incorporation of DIB. 
Grafting onto DIB homopolymers has been achieved by reacting polystyryl anions with the substrate for an extended time period $(2-12 \mathrm{~h})$ at $35^{\circ} \mathrm{C}$ in the presence of THF, and the graft polymers obtained had relatively narrow MWDs (PDI $=1.21-1.24) .{ }^{8}$ Grafting from $\alpha$ methylstyrene-DIB copolymers by activation of the pendent isopropenyl moieties with $s$-BuLi and subsequent addition of styrene was also demonstrated, although the graft polymers obtained had broader MWDs (PDI $=1.2-2.5){ }^{8}$

Three types of unsaturated species (1-3) and three types of propagating centers (4-6) are present in the copolymerization of styrene and DIB (Scheme S-1). Following the initiation step, the reaction of the two monomers is therefore best described as a terpolymerization. Keeping in mind the complexity of the copolymerization reaction and the potential influence of solvency conditions and temperature, the relative reactivities indicated in Scheme S-1 are proposed for unsaturated species 1-3 and propagating centers 4-6.

\section{Scheme S-1. Reactivity of Unsaturated Species and Propagating Centers}

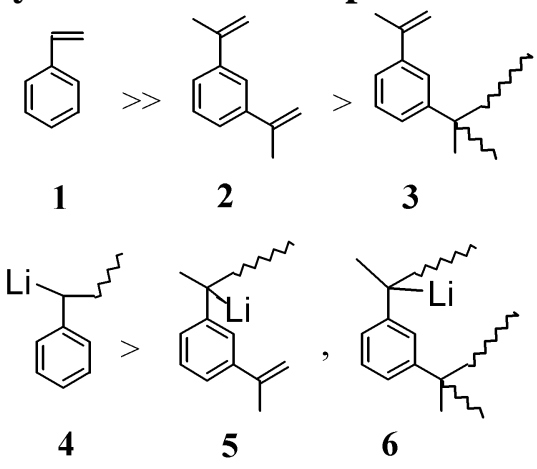

Initiation. The copolymerization of styrene and DIB was initiated with oligostyryllithium $\left(X_{\mathrm{n}}=\right.$ 5) rather than $s-\mathrm{BuLi}$, to minimize the reaction of both double bonds of DIB. Because of the apparent lack of selectivity of $s-\mathrm{BuLi}$ in the initiation reaction, direct slow addition of the styrene-DIB monomer mixture to $s$-BuLi may lead to a mixture of mainly linear polymers in a 2:1 molecular weight ratio, due to the simultaneous formation of mono- and dilithiated species (and possibly a small amount of lithiated oligomers). SEC elution curves obtained for linear copolymers synthesized under different conditions are compared in Figure S-1, and the characterization results are summarized in Table S-1. Samples were removed from the reactor and terminated at predetermined time intervals after monomer addition was completed $(5,60$, and $120 \mathrm{~min}$ ). Only the results obtained for a waiting time of $5 \mathrm{~min}$ will be discussed initially, and the influence of post-addition polymerization time on the reaction will be discussed later.

The polymer obtained by direct initiation with $s$-BuLi (Figure S-1, curve a) has two relatively well-resolved peaks, the leftmost peak being centered at an apparent (polystyrene-equivalent) molecular weight of 13800 while the rightmost peak is centered around 6500. The target molecular weight of the polymer, corresponding to $X_{\mathrm{n}}=55$, was $M_{\mathrm{n}}=6460$. Because of the almost exact 2:1 molecular weight ratio for the two peaks, the leftmost peak is attributed to the reaction of both isopropenyl moieties on some of the DIB molecules with $s$-BuLi, and subsequent initiation by the dilithiated species to yield a linear 'dimer' with a molecular weight twice as high. Dimer formation should be less likely when oligostyryllithium is generated prior to the mixed monomer addition, because of its lower reactivity (and higher selectivity relative to $s$-BuLi) towards the first isopropenyl group of DIB. 
It should be noted that for the purpose of determining the reaction conditions necessary to minimize cross-linking reactions, it is irrelevant whether apparent or absolute SEC characterization data $\left(M_{\mathrm{n}}, \mathrm{PDI}\right)$ are used. For the characterization data reported in Table S-1, for example, apparent (polystyrene equivalent) results were preferred over absolute (MALLS) results. The signal from the MALLS detector was very noisy and deemed unreliable, due to the low molecular weight of the samples $\left(M_{\mathrm{n}}=6000-9000\right)$. In general, it was found that when apparent and absolute characterization data were both available for the samples, the same trends were present among each series of results. Consequently, conclusions regarding reaction condition optimization could be drawn interchangeably from either apparent or absolute SEC characterization data.

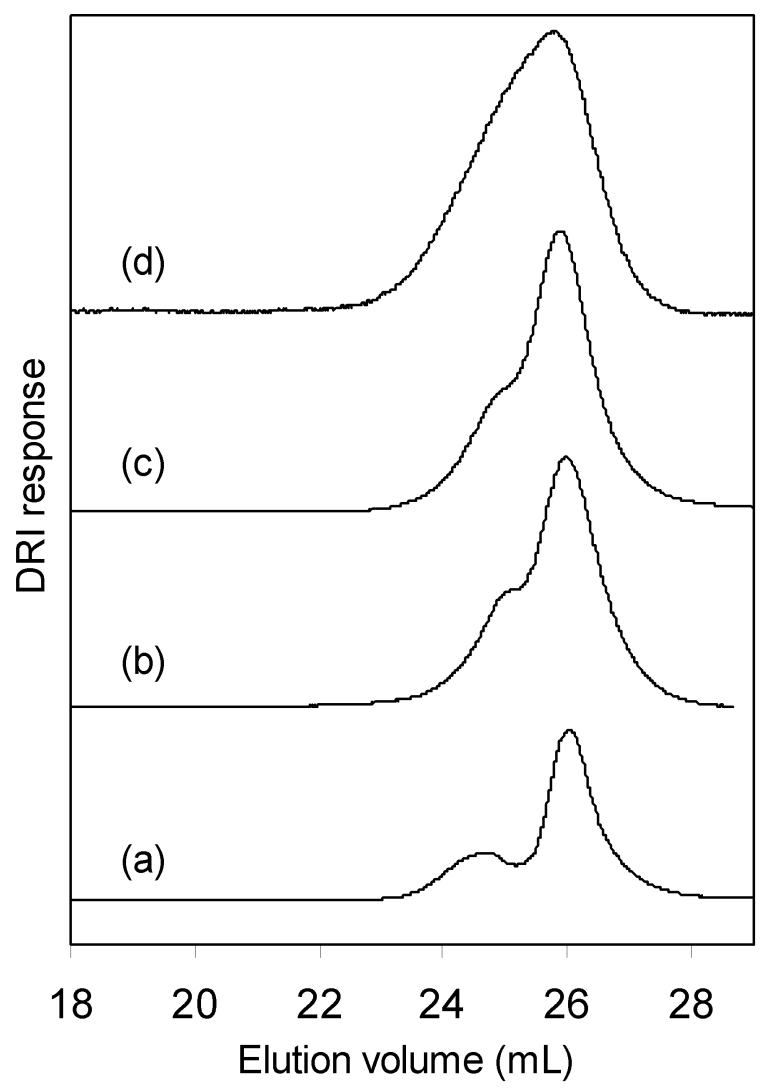

Figure S-1. Influence of monomer addition rate and protocol on the SEC elution curves of linear styrene-DIB copolymers after 5 min post-addition polymerization time: (a) direct $s$-BuLi initiation; (b) semibatch addition over $13 \mathrm{~min}$ (L-5); (c) dropwise addition over $16 \mathrm{~min}$ (L-2); (d) dropwise addition over $24 \mathrm{~min}$ (L-3).

Control of the comonomer addition rate was important from two viewpoints: 1) To guarantee a uniform distribution of DIB units along the chain, and 2) to ensure that the living chains were capped with a DIB unit most of the time, so as to minimize cross-linking reactions. To achieve a uniform distribution of branching points in the one-pot process, monomers $\mathbf{1}$ and $\mathbf{2}$ should ideally copolymerize randomly to full conversion and without any reaction of species $\mathbf{3}$, but this is unfortunately not the case. In an investigation of the copolymerization behavior of styrene-DIB mixtures, ${ }^{7}$ a large reactivity difference between styrene and DIB was inferred on the basis of the 
color changes observed, and confirmed by composition analysis of the copolymers obtained. Styrene thus polymerized first with a characteristic yellow color, samples removed from the reactor at low conversion being devoid of unsaturation. DIB then polymerized slowly with a characteristic dark brown coloration. The sequential copolymerization behavior of the two monomers was likewise obvious in the semibatch addition protocol used in this work: Each time an aliquot of monomer mixture was added to the reaction, a very fast color transition from dark brown to yellow (styrene polymerization) was observed, and followed by another transition back to dark brown (DIB polymerization) after a few seconds. The homopolymerization of styrene in THF being very fast, it is consumed quickly. The gradual change to a dark brown color observed after a few seconds corresponds to the formation of the DIB anions. Since there is an 80-sec delay between each aliquot addition, the living chains remain capped by a DIB unit most of the time, as demonstrated by the stable brown coloration of the solution over that time interval.

Another problem specific to the one-pot process is that if the conversion of DIB is incomplete, one or both double bonds of the residual monomer could react upon addition of $s$ $\mathrm{BuLi}$ in the activation step leading to the graft polymer of the following generation, and produce linear polymer contaminant. The reaction of unsaturated species 3, causing cross-linking, is also favored at high conversion. ${ }^{9}$ To minimize these problems, the conditions used were optimized in terms of reaction temperature, monomer addition protocol, polymerization time allowed after monomer addition, styrene:DIB monomer ratio, and polymer concentration in the reaction. The influence of some of these factors on the course of the reaction is discussed below.

Due to the potential formation of cross-linked species in the presence of DIB units, the reproducibility of the reactions reported is necessarily lower than for typical anionic polymerization reactions. To compensate for this, many reactions (in particular those using the different monomer addition protocols) were repeated multiple times to allow the calculation of average PDI and standard deviation values as a measure of reproducibility. The SEC elution curves presented in the figures are typical results obtained for the different reaction conditions selected.

Monomer Addition Protocol. As discussed above, styrene and DIB display significant reactivity differences. If the monomer mixture were added too fast either a tapered block copolymer, with styrene-rich and DIB-rich segments, or a diblock copolymer could be generated, depending on the rate of monomer addition relative to the rate of propagation. Two different monomer addition protocols were explored to achieve a more uniform distribution of grafting sites along the substrate. In each case the monomer was added to the reaction in successive steps, each addition being followed by a short waiting time. The first approach used dropwise addition of the monomer mixture until the yellow coloration of polystyryl anions was observed; the second method was the semibatch addition of fixed volume aliquots of monomer mixture as described in the Experimental Section.

A comparison of curves $c$ (sample L-2) and b (sample L-5) in Figure S-1 shows that dropwise and semibatch additions in fact yielded very similar results for the linear copolymer. This is confirmed when comparing the results summarized in Table S-1 for samples L-2 and L-5, for which the monomer was added over a similar time interval (13-16 min). The reproducibility of the dropwise and semibatch addition protocols was verified by calculating the average PDI and standard deviation (SD) on the PDI obtained for a series of reactions with a styrene:DIB mole ratio of 3 , for samples removed 5 min after completing monomer addition. For dropwise addition, an average $\mathrm{PDI}=1.4_{4}$ and $\mathrm{SD}=0.15$ were obtained for a series of 5 experiments. For 
semibatch addition, an average $\mathrm{PDI}=1.36$ and $\mathrm{SD}=0.06$ were obtained over 9 runs. For samples removed from the reactor $1 \mathrm{~h}$ after monomer addition (corresponding to the preferred conditions), dropwise addition yielded an average PDI $=1.5_{7}$ and SD $=0.17$ (8 runs), as compared with an average PDI $=1.42$ and $\mathrm{SD}=0.07$ ( 9 runs) for semibatch addition. The reproducibility statistics show that semibatch addition yields insignificantly lower PDI values for the linear copolymer (for both $5 \mathrm{~min}$ and $1 \mathrm{~h}$ post-addition polymerization times), but the variations in PDI are significantly lower. The influence of the addition protocol is more noticeable in the synthesis of the G0 copolymers.

Slower monomer addition may favor a higher DIB conversion, but it also causes broadening of the MWD. Thus increasing the manual monomer addition time from 16 min (Figure S-1c, sample L-2) to $24 \mathrm{~min}$ (Figure S-1d, sample L-3) led to an increase in the number-average molecular weight $\left(M_{\mathrm{n}}\right)$ and PDI $\left(M_{\mathrm{w}} / M_{\mathrm{n}}\right)$. If the monomer mixture is added very slowly, a higher average concentration of polystyryl anions may be present throughout the reaction. The higher reactivity of polystyryl anions relative to more hindered polyDIB anions should increase the probability of pendent isopropenyl group attack, causing broadening of the MWD. Gelation of the reaction mixture was indeed observed when the monomer addition time was increased to 45 $\min$.

Even when using the dropwise or semibatch monomer addition protocols with a short overall addition time, it appears impossible to avoid side reactions altogether: Shoulders are clearly observable on curves (b) and (c) of Figure S-1, and are attributed to attack of the polystyryl anions on pendent isopropenyl groups to form three-arm stars (and possibly higher order polycondensates, alternately identified as cross-linked material). For curve (b), for example, the main peak is observed at an apparent molecular weight of 6700, while the shoulder is located around 10200 and not as well resolved from the main peak as in curve (a). The molecular weight ratio for the two species (1.5), significantly lower than 2, is consistent with the formation of cross-linked species rather than linear polymer. Notwithstanding the low molecular weight ratio inconsistent with the formation of two distinct linear polymeric species, an alternate explanation for the presence of a shoulder in the SEC curves could be the incomplete consumption of $s$-BuLi in the synthesis of the oligostyryllithium initiator, causing dilithiation of the DIB added. To verify this hypothesis the amount of styrene used was doubled, to obtain oligostyryllithium with $X_{\mathrm{n}}=10$. A significant decrease in PDI would be expected under these conditions, if the shoulder were linked to the presence of residual $s$-BuLi. The copolymer obtained with semibatch addition and a one-hour post-addition polymerization time had a PDI $=1.35$, very similar to the average $\mathrm{PDI}=1.4_{2} \pm 0.07$ obtained using the $X_{\mathrm{n}}=5$ oligomers. This confirms that the shoulder is not due to residual $s$-BuLi.

Reaction Temperature. The temperature affects both the polymerization rate and the attainable monomer conversion. Following the synthesis of the oligostryryllithium initiator, each monomer addition can be approximated (on average) as one cross-propagation step of DIB anions with styrene, two styrene homopolymerization steps, and one cross-propagation step of oligostyryl anions with DIB in the presence of THF. There is no hindrance to the full conversion of styrene, since both styryl ${ }^{10}$ and $\alpha$-methylstyryl ${ }^{11}$ anions react rapidly with styrene. The fast crossover reaction of DIB anions with styrene is also confirmed by the rapid color change from brown to yellow observed upon addition of the monomer mixture. The cross-propagation reaction of polystyryl anions with DIB and the homopolymerization of DIB are much slower, ${ }^{8}$ so unreacted DIB may have the tendency to accumulate in the reaction. Since the homopolymerization of DIB 
is reversible, a low temperature should favor a high conversion (in the event that DIB does accumulate in the reactor), albeit there are no reports on the temperature necessary to achieve this. In the polymerization of $\alpha$-methylstyrene, with a ceiling temperature below $25^{\circ} \mathrm{C}$, a high conversion can be attained only at very low temperatures (e.g. $\left.-80{ }^{\circ} \mathrm{C}\right) .{ }^{12,13}$

The reaction temperature may also affect the propagation rate. For many anionic polymerization reactions the apparent activation energy for the propagation rate constant is typically very low. The rate of anionic polymerization of $\alpha$-methylstyrene was thus reported to be only about 4 times lower at $-7{ }^{\circ} \mathrm{C}$ than at $-10{ }^{\circ} \mathrm{C} .{ }^{13}$ The relative insensitivity of the polymerization rate to temperature in these reactions is consistent with apparent activation energies close to zero. The rate of homopolymerization of DIB and 1,4-DIB is rather slow, the conversion attained in THF after $120 \mathrm{~min}$ at $-30{ }^{\circ} \mathrm{C}$ being only $93 \%$ and $65 \%$ for DIB and $1,4-$ DIB, respectively. ${ }^{8}$ Unfortunately, rate information is unavailable for the styrene-DIB copolymerization. We found by $\mathrm{GC}$ analysis of the reaction mixtures that at low temperature ($78^{\circ} \mathrm{C}$ ) the polymerization rates were somewhat lower than at $-35^{\circ} \mathrm{C}$ both for the homo- and copolymerization of DIB.

On the basis of the above considerations, a temperature of $-78{ }^{\circ} \mathrm{C}$ was selected for the synthesis of the styrene-DIB copolymers.

Polymer Concentration. Because a significant PDI increase is observed in the synthesis of the G0 copolymers, it may be preferable to conduct the reaction at a lower concentration to reduce the probability of intermolecular cross-link formation. Characterization results are provided in Table 2 for samples G0-5a and G0-5b. Both samples were synthesized from the same linear precursor (L-5), which was divided into two portions and diluted with different amounts of solvent (200 mL and $300 \mathrm{~mL}$, respectively). A somewhat lower PDI was obtained at the lower concentration (sample G0-5b). Analysis of the reaction mixture by GC showed that the DIB conversion attained $1 \mathrm{~h}$ after complete monomer addition was comparable at both concentrations. On the basis of these limited results, it was concluded that dilution favors a somewhat lower PDI without affecting DIB conversion.

Effects of Lithium Salt Modifiers. Lithium chloride and lithium alcoholates are widely used as additives to modify the reactivity of anionic propagating centers when lithium is the counterion. ${ }^{14,15}$ These compounds could be useful to fine-tune the reactivity of the ionic species present in the reaction, and increase their selectivity towards each double bond of DIB to avoid side reactions. The results obtained using $\mathrm{LiCl}$ and $\mathrm{LiOMe}$ in the styrene-DIB copolymerization reaction were disappointing: When these salts were added in a 6:1 ratio relative to the living ends, no significant improvement in PDI was observed for the linear copolymers (average PDI $=$ $1.34 \pm 0.10$ over 6 runs) nor for the G0 copolymer (average PDI $=1.78 \pm 0.10$ ).

1,4-Diisopropenylbenzene (1,4-DIB) in Replacement of DIB. Because the reactivity difference between the two isopropenyl functionalities of DIB is apparently not sufficiently large, some dimerization and multimerization is invariably observed in the synthesis of the 'linear' and G0 styrene-DIB copolymers. In the hope of minimizing these side reactions, 1,4-DIB was investigated to replace (1,3-)DIB in the reactions. Since the isopropenyl moieties of 1,4-DIB are in the para-positions of the phenyl ring, stronger conjugation should make the first double bond of 1,4-DIB more reactive than in DIB. The pendent isopropenyl moiety should also be more subjected to electron donation by the saturated polymer backbone once the first isopropenyl 
moiety has reacted, making it less susceptible to cross-linking. In other words, the two isopropenyl groups in 1,4-DIB are expected to display a larger reactivity difference than in DIB.

The results obtained for a styrene:1,4-DIB ratio of 3 , using a semibatch addition protocol and a 1-h post-addition polymerization time, were in fact very similar to those obtained for DIB, with an average PDI value of 1.42 and $\mathrm{SD}=0.10$ over 3 runs for the linear copolymer (for DIB, PDI $=1.42 \pm 0.07$ ), and an average $\mathrm{PDI}=1.6_{7}$ and $\mathrm{SD}=0.22$ for the $\mathrm{G} 0$ copolymer also over 3 runs (for DIB, PDI $=1.8_{1} \pm 0.13$ ). Different hypotheses can be suggested for the lack of improvement in PDI when using 1,4-DIB. The polystyryl anions may be too reactive to differentiate among the two isopropenyl groups of 1,4-DIB. Another possibility is the poor solubility of 1,4-DIB in THF at low temperature: When the monomer mixture was added at $-78^{\circ} \mathrm{C}, 1,4-\mathrm{DIB}$ (in contrast to the 1,3-isomer) tended to crystallize initially on the surface of the solution, making it temporarily unavailable for the reaction. Finally, because the pendent isopropenyl groups of the (1,3-)DIB copolymers are more hindered by the backbone chain than in the 1,4-DIB copolymers, the pendent isopropenyl moieties in DIB units may actually be less reactive toward the macroanions than those in 1,4-DIB units.

Synthesis of Higher Generation DIB Copolymers. The synthesis of G1 styrene-DIB copolymers (as precursors for the synthesis of G2 polymers) by the one-pot method has been achieved only with limited success: Residual DIB monomer in the G0 substrate synthesis invariably produced significant amounts of linear copolymer. In such an attempt with a target $M_{\mathrm{n}}$ $=5000$ for the linear copolymer, $M_{\mathrm{n}}=5000$ for the G0 side chains, and $M_{\mathrm{n}}=3000$ for the G1 side chains, the copolymer obtained (apparent $M_{\mathrm{w}}=423000, M_{\mathrm{w}} / M_{\mathrm{n}}=1.84$ ) was contaminated with $20 \%$ linear copolymer. Using the impure G1 copolymer sample to synthesize a G2 polymer would give a mixture of $\mathrm{G} 2, \mathrm{G} 0$, and linear components. In an attempt to avoid this problem the G1 styrene-DIB copolymer was purified by fractionation, to remove unreacted DIB and linear copolymer. A G1 substrate (target side chain $M_{\mathrm{n}}=5000$ at every step) was also synthesized using a G0 styrene-DIB copolymer precipitated to remove residual DIB (apparent $M_{\mathrm{w}}=670000$, $\left.M_{\mathrm{w}} / M_{\mathrm{n}}=1.95\right)$. While these procedures are no longer true one-pot processes, activation of the $\mathrm{G} 1$ copolymer under these conditions should at least allow the synthesis of G2 polymers with minimal contamination. Activation times of $6-10 \mathrm{~h}$ with a $20 \%$ excess of $s$-BuLi were used, and styrene monomer was added to the G1 substrate for a target side chain $M_{\mathrm{n}}=2500$. In all cases a gel formed within a few minutes of styrene addition (1-2 min without $\mathrm{LiCl}$, ca. 4 min with $\mathrm{LiCl}$ ), demonstrating the presence of residual isopropenyl groups on the substrate in spite of the excess of activating compound and the long activation times used.

Side Chain Extension Reactions with Copolymer Isolation. Elution curves are compared in Figure 5 for the fractionated G1 copolymers obtained by successive monomer additions after isolation of the copolymer. The corresponding curves for the non-fractionated (crude) products are provided in Figure S-2. It is clear that the rightmost peak, due to the linear contaminant, is narrow and monomodal in all cases. This confirms that the linear component originates exclusively from the excess of $s$-BuLi used to activate the pendent double bonds on the copolymer substrate. If residual DIB were present in the reaction a bimodal distribution (as observed in Figure 4a) would be expected, due to the activation of one or both double bonds of the residual DIB by $s$-BuLi. 


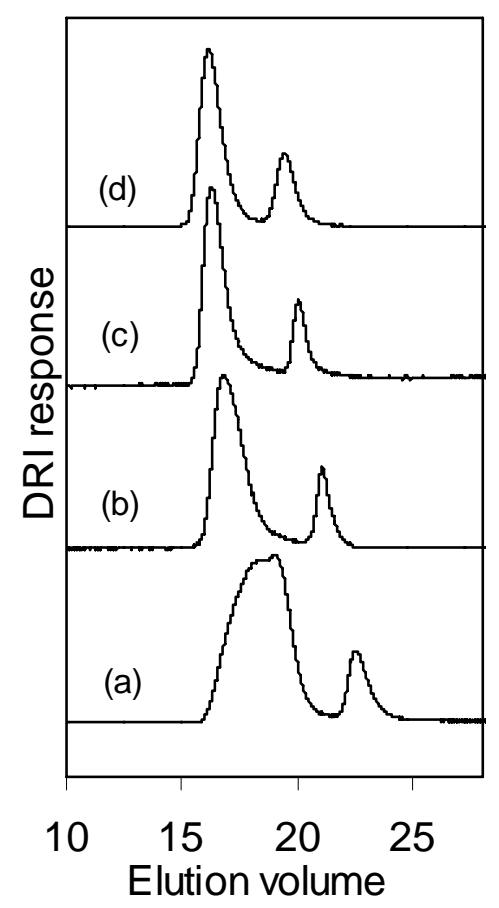

Figure S-2. SEC elution curves for the crude products in the synthesis of analogous G1 polystyrenes (after copolymer isolation) described in Table 4: (a) $M_{\mathrm{n}}^{\mathrm{SC}}=2500$, (b) $M_{\mathrm{n}}^{\mathrm{SC}}=5000$, (c) $M_{\mathrm{n}}^{\mathrm{SC}}=10000,(\mathrm{~d}) M_{\mathrm{n}}^{\mathrm{SC}}=20000$.

\section{References}

(1) (a) Kee, R. A.; Gauthier, M. Macromolecules 2002, 35, 6526. (b) Gauthier, M.; Li, J.; Dockendorff, J. Macromolecules 2003, 36, 2642.

(2) Mitin, Y. V. Zhur. Obshchei Khim. 1958, 28, 3302. Chem. Abstr. 1959, 53, 77647.

(3) Lutz, P.; Beinert, G.; Franta, E.; Rempp, P. Eur. Polym. J. 1979, 15, 1111.

(4) Lipton, M. F.; Sorensen, C. M.; Sadler, A. C.; Shapiro, R. H. J. Organomet. Chem. 1980, 186, 155.

(5) Baker, J. R. J. Appl. Polym. Sci. 1971, 15, 411.

(6) Gauthier, M.; Möller, M. Macromolecules 1991, 24, 4548.

(7) Yu, Y. S.; Dubois, P.; Jérôme, R.; Teyssié, P. Macromolecules 1996, 29, 1753.

(8) Lutz, P.; Beinert, G.; Rempp, P. Makromol. Chem. 1982, 183, 2787.

(9) Naraghi, K. S.; Meneghetti, S. P.; Lutz, P. J. Macromol. Rapid Commun. 1999, $20,122$.

(10) Lee, C. L.; Smid, J.; Szwarc, M. J. Am. Chem. Soc. 1961, 83, 2961.

(11) Bhattacharyya, D. N.; Lee, C. L.; Smid, J.; Szwarc, M. J. Am. Chem. Soc. 1963, 85, 533.

(12) Worsfold, D. J.; Bywater, S. J. Polym. Sci. 1957, 26, 299.

(13) Worsfold, D. J.; Bywater, S. Can. J. Chem. 1958, 36, 1141.

(14) Huyskens, P. L.; Verheyden, H.; Van Lierde, P. J. Mol. Liquids 1998, 78, 151.

(15) Yakimansky, A. V.; Müller, A. H. E.; Van Beylen, M. Macromolecules 2000, 33, 5686. 
Table S-1. Optimization of Reaction Conditions for the Synthesis of Linear Styrene-DIB Copolymers $^{\mathrm{a}}$

\begin{tabular}{|c|c|c|c|c|c|c|}
\hline \multirow[t]{2}{*}{ Sample } & \multirow[t]{2}{*}{ St:DIB ${ }^{b}$} & \multicolumn{2}{|c|}{ Monomer addition } & \multirow{2}{*}{$\begin{array}{l}\text { Polym. time } \\
\text { /min }\end{array}$} & \multicolumn{2}{|c|}{ Polymer ${ }^{\mathrm{d}}$} \\
\hline & & Method & $\begin{array}{l}\text { Time } \\
/ \mathrm{min}\end{array}$ & & $\begin{array}{c}M_{\mathrm{n}} \\
/ 10^{3}\end{array}$ & $M_{\mathrm{w}} / M_{\mathrm{n}}$ \\
\hline \multirow[t]{2}{*}{ L-2 } & \multirow[t]{2}{*}{3} & Drop- & \multirow[t]{2}{*}{16} & 5 & 6.2 & 1.30 \\
\hline & & wise & & 60 & 7.7 & 1.38 \\
\hline \multirow[t]{3}{*}{ L-3 } & \multirow[t]{3}{*}{3} & Drop- & \multirow[t]{3}{*}{24} & 5 & 7.3 & 1.40 \\
\hline & & wise & & 60 & 8.0 & 1.49 \\
\hline & & & & 120 & 9.3 & 1.69 \\
\hline \multirow[t]{2}{*}{ L-5 } & \multirow[t]{2}{*}{3} & Semi- & \multirow[t]{2}{*}{13} & 5 & 6.8 & 1.27 \\
\hline & & batch & & 60 & 7.5 & 1.32 \\
\hline \multirow[t]{2}{*}{ L-6 } & \multirow[t]{2}{*}{2.5} & Drop- & \multirow[t]{2}{*}{16} & 5 & 6.1 & 1.41 \\
\hline & & wise & & 60 & 7.8 & 1.62 \\
\hline \multirow[t]{2}{*}{ L-7 } & \multirow[t]{2}{*}{2.5} & Semi- & \multirow[t]{2}{*}{17} & 5 & 6.1 & 1.21 \\
\hline & & batch & & 60 & 7.8 & 1.43 \\
\hline \multirow[t]{2}{*}{ L-8 } & \multirow[t]{2}{*}{3.5} & Semi- & \multirow[t]{2}{*}{12} & 5 & 6.3 & 1.35 \\
\hline & & batch & & 60 & 7.4 & 1.48 \\
\hline
\end{tabular}

${ }^{\text {a }} X_{\mathrm{n}}=5$ oligostyryllithium as initiator, 50 equiv mixed monomer added for chain growth at $\mathrm{T}=-78{ }^{\circ} \mathrm{C} .{ }^{\mathrm{b}}$ Styrene:DIB monomer ratio used in the reaction. ${ }^{\mathrm{c}}$ Polymerization time allowed after completing monomer addition. ${ }^{\mathrm{d}}$ Apparent values based on linear polystyrene calibration. 